\title{
$\mathbb{N}<\mathrm{T}$
}

Karlsruhe Institute of Technology

\section{Data Management and Analysis at the Large Scale Data Facility}

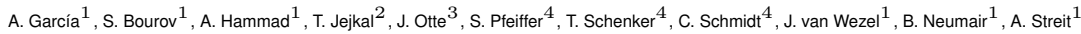

1 Steinbuch Centre for Computing, KIT

2 Institute for Data Processing and Electronics, KIT

3 Institute of Toxicology and Genetics, KIT

${ }^{4}$ Institute for Applied Computer Science, KIT

ICDIM 2011, Melbourne | September 27, 2011

\section{KARLSRUHE INSTITUTE OF TECHNOLOGY}

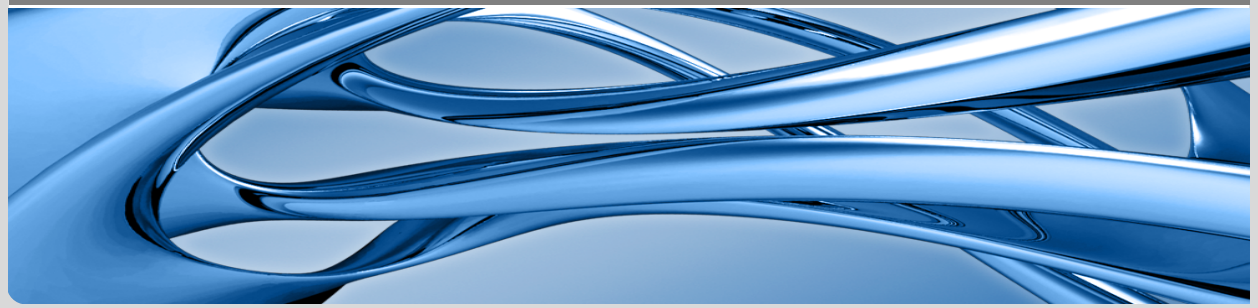




\section{Executive summary}

- Many experiments have a data-management problem!

- This project aims at improving the situation, with adapted infrastructure and services

- Data Intensive Computing workflows are critical for the value of the data

- We present a concrete data intensive analysis use case at the LSDF 


\section{What is the data challenge?}

\section{Science produces data!}

- Experiments getting exponentially more data

$\Rightarrow$ experiments need storage

$\Rightarrow$ need sophisticated data analysis workflows

- Old data is very valuable

a to analyse change in time

- for reprocessing

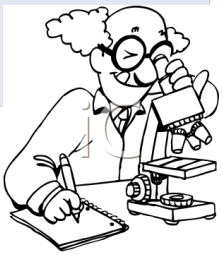

- for analysis by other scientists, in other contexts

a Invisible (not-found, no-metadata) data is lost data

$\Rightarrow$ adequate meta data greatly increases data value

$\Rightarrow$ single big scientific DB is more valuable than

many small ones

- Data is used by large virtual communities!

$\Rightarrow$ access to data is critical 


\section{What is the data challenge?}

\section{Science produces data!}

- Experiments getting exponentially more data

$\Rightarrow$ experiments need storage

$\Rightarrow$ need sophisticated data analysis workflows

- Old data is very valuable

- to analyse change in time

- for reprocessing

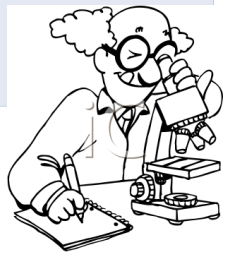

- for analysis by other scientists, in other contexts

Invisible

data is lost data

$\Rightarrow$ adequate meta data greatly increases data value

$\Rightarrow$ single big scientific DB is more valuable than

many small ones

- Data is used by large virtual communities!

$\Rightarrow$ access to data is critical 


\section{What is the data challenge?}

\section{Science produces data!}

- Experiments getting exponentially more data

$\Rightarrow$ experiments need storage

$\Rightarrow$ need sophisticated data analysis workflows

- Old data is very valuable

- to analyse change in time

- for reprocessing

- for analysis by other scientists, in other contexts

- Invisible (not-found, no-metadata) data is lost data

$\Rightarrow$ adequate meta data greatly increases data value

$\Rightarrow$ single big scientific DB is more valuable than many small ones

- Data is used by large virtual communities!

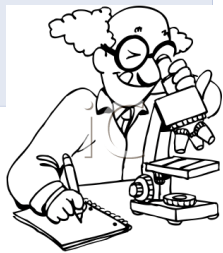

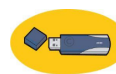

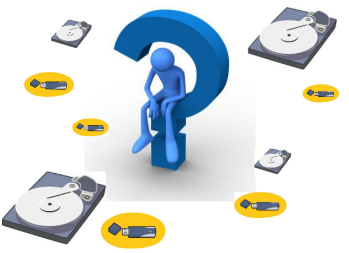

$\Rightarrow$ access to data is critical 


\section{What is the data challenge?}

\section{Science produces data!}

- Experiments getting exponentially more data

$\Rightarrow$ experiments need storage

$\Rightarrow$ need sophisticated data analysis workflows

- Old data is very valuable

- to analyse change in time

- for reprocessing

- for analysis by other scientists, in other contexts

- Invisible (not-found, no-metadata) data is lost data

$\Rightarrow$ adequate meta data greatly increases data value

$\Rightarrow$ single big scientific DB is more valuable than many small ones

- Data is used by large virtual communities!

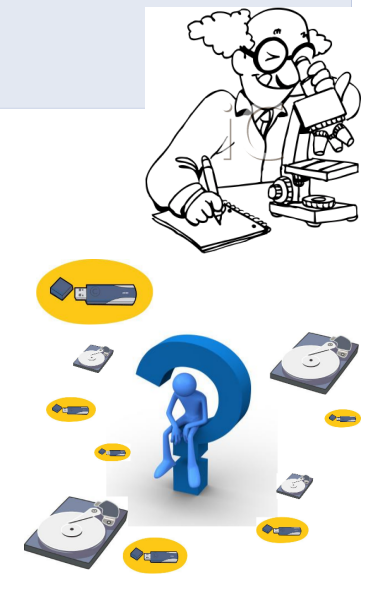

$\Rightarrow$ access to data is critical 


\section{Why do experiments produce so much data?}

Zebrafish embryo, raw picture, 4MB (24 per fish) 


\section{Why do experiments produce so much data?}

- Institute of Toxicology and Genetics @ KIT

$\Rightarrow$ Zebra fishes' embryonal development reconstruction

$\Rightarrow$ Toxicological studies of drugs

- High Throughput Microscopy

- fully automated microscopes

- robot moves object to microscope

- can potentially run $24^{\star} 7$

- produce high resolution images (4 MB each)

- over varying parameters (focus point, cell markers, ...) 


\section{Why do experiments produce so much data?}

- Institute of Toxicology and Genetics @ KIT

$\Rightarrow$ Zebra fishes' embryonal development reconstruction

$\Rightarrow$ Toxicological studies of drugs

- High Throughput Microscopy

- fully automated microscopes

- robot moves object to microscope

- can potentially run $24^{\star} 7$

- produce high resolution images (4 MB each)

- over varying parameters (focus point, cell markers, ...)

- $\approx 200 \mathrm{k}$ images per day, $\approx 2 \mathrm{~TB} /$ day

- Estimated: $1+\mathrm{PB} /$ year in 2012, $6 \mathrm{~PB} / \mathrm{year}$ in 2014

- Raw data must be heavily analysed

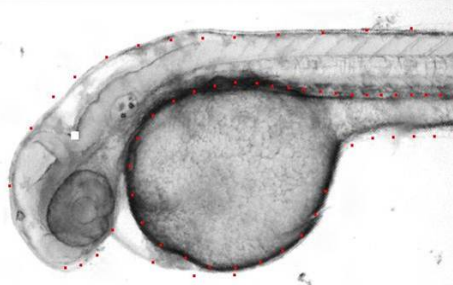




\section{The Large Scale Data Facility Project}

\section{LSDF started end of 2009 at KIT}

- involving several institutes

- in cooperation with BioQuant of Univ. Heidelberg 


\section{The Large Scale Data Facility Project}

\section{LSDF started end of 2009 at KIT}

- involving several institutes

- in cooperation with BioQuant of Univ. Heidelberg

- to address the needs of Data Intensive Science:

- providing large scale storage and data processing

- open protocols and APIs for access to data and metadata

- added value services for community specific needs (multi-disciplinary) 


\section{What infrastructure are we talking about?}



- Currently 2 PB in 2 storage systems

- Tape for archive and backup

- Dedicated 10 GE network backbone

- Direct network connection to some institutes

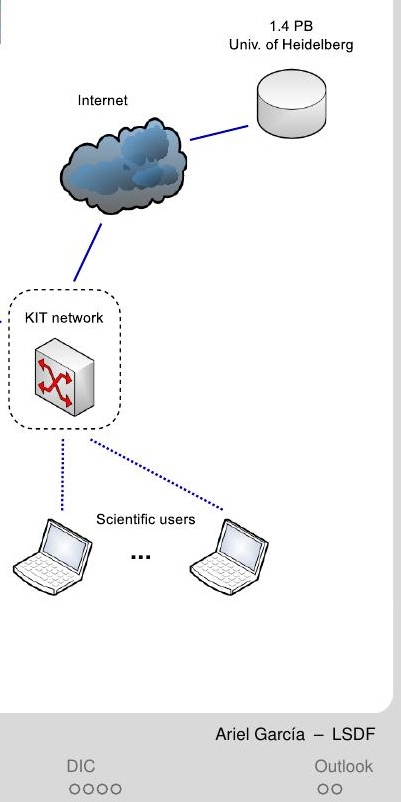




\section{The current architecture}

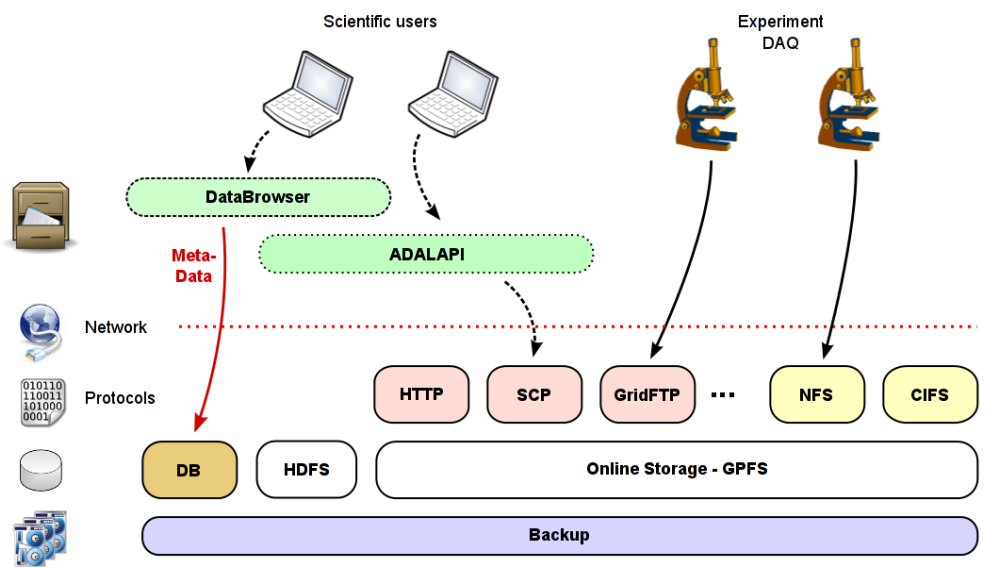




\section{How to deal with so much data?}

- Metadata is essential

- Needs to be stored and kept up to date with data

- Metadata schema is highly project-dependent

$\Rightarrow$ use a project metadata DB

Example: 


\section{How to deal with so much data?}

- Metadata is essential

- Needs to be stored and kept up to date with data

- Metadata schema is highly project-dependent

$\Rightarrow$ use a project metadata DB

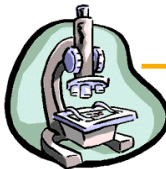

Experiment

\section{DATA}

- write once, read many - persistent
METADATA 1

- basic metadata

- processing A metadata

- results $A$

\section{METADATA 2}

- basic metadata

- processing B1 metadata

- results B1

- processing B2 metadata - results B2

Example:

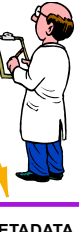

BASIC METADATA

- write once, read many

- persistent

\section{METADATA N}

- basic metadata

- processing $\mathrm{N}$ metadata - results $N$ 


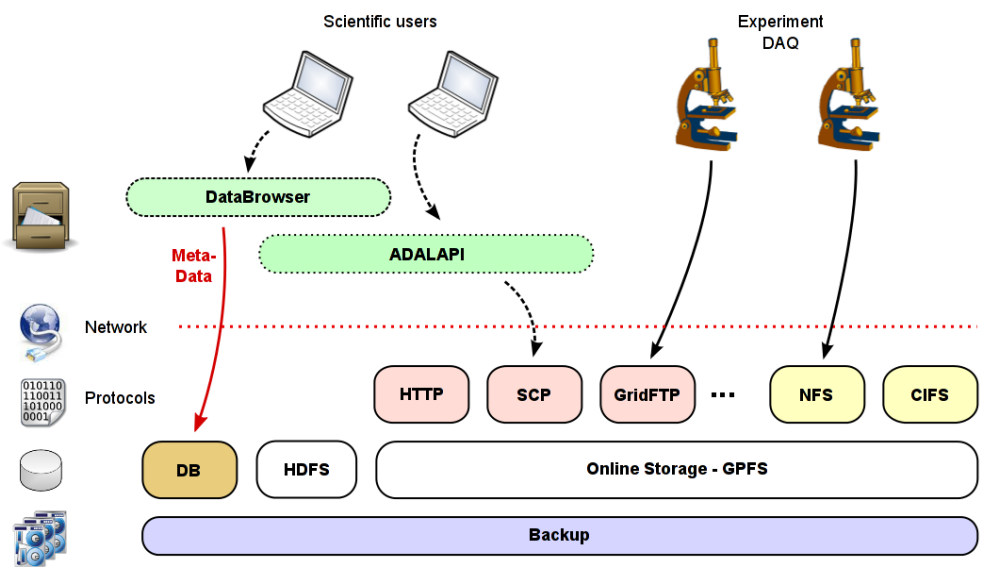




\section{Which access APIs and tools?}

- Different components accessible through different protocols and APIs

$\Rightarrow$ need a unified access layer

- Abstract Data Access Layer, low-level interface to LSDF

$\Rightarrow$ extensible to support new backends, authentication mechanisms

a For end-users: DataBrowser

- graphical tool for exploring and managing the LSDF data

- also library for meta-data access

a based on ADALAP|

- connects to the meta-data repository

- will be available as web GUI 


\section{Which access APIs and tools?}

- Different components accessible through different protocols and APIs

$\Rightarrow$ need a unified access layer

- Abstract Data Access Layer, low-level interface to LSDF

$\Rightarrow$ extensible to support new backends, authentication mechanisms

- For end-users: DataBrowser

- graphical tool for exploring and managing the LSDF data

- also library for meta-data access

- based on ADALAPI

- connects to the meta-data repository

- will be available as web GUI

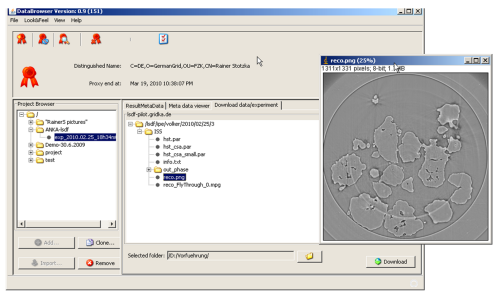




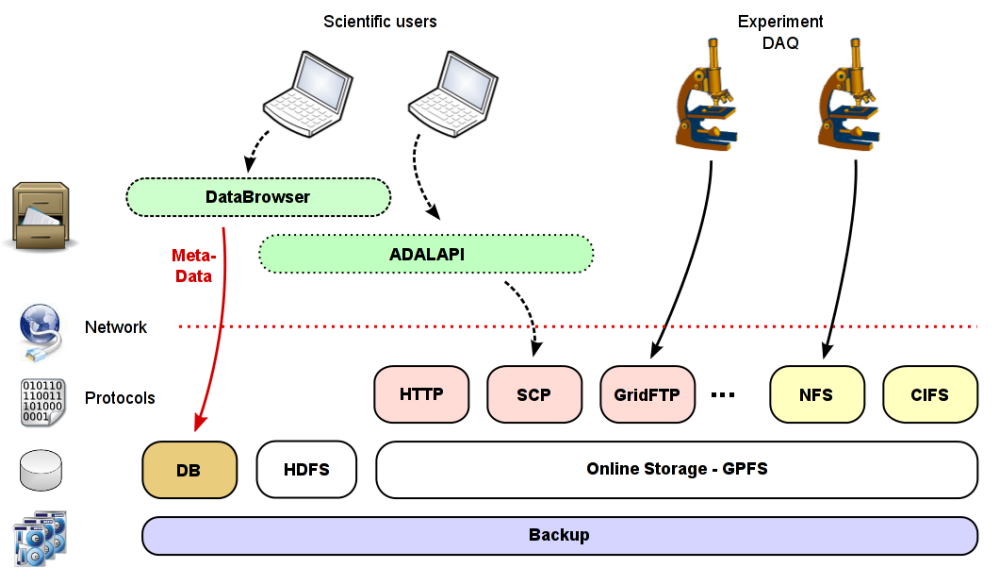




\section{Can we process the data?}

- Data has to be processed!

- Exascale $\Rightarrow$ bring computing to the data!! (15 days to transfer 1 PB over ideal 10Gb/s link)

$\Rightarrow$ dedicated 60 nodes cluster

- users can deploy own dedicated

data-processing VMs

- reliable, highly flexible,

and very fast to deploy 


\section{Can we process the data?}

- Data has to be processed!

- Exascale $\Rightarrow$ bring computing to the data!! (15 days to transfer 1 PB over ideal 10Gb/s link)

$\Rightarrow$ dedicated 60 nodes cluster

- Hadoop environment

+ 110 TB Hadoop filesystem

- extreme scalability

a Cloud environment OpenNebula

a users can deploy own dedicated data-processing VMs

a reliable, highly flexible, and very fast to deploy

:

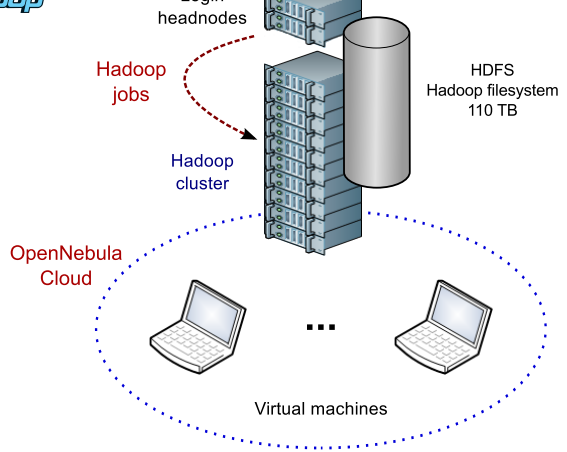




\section{Can we process the data?}

- Data has to be processed!

- Exascale $\Rightarrow$ bring computing to the data!! (15 days to transfer 1 PB over ideal 10Gb/s link)

$\Rightarrow$ dedicated 60 nodes cluster

- Hadoop environment

+ 110 TB Hadoop filesystem

- extreme scalability

- Cloud environment OpenNebula

\section{OpenNebula.org}

- users can deploy own dedicated data-processing VMs

- reliable, highly flexible, and very fast to deploy

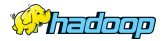

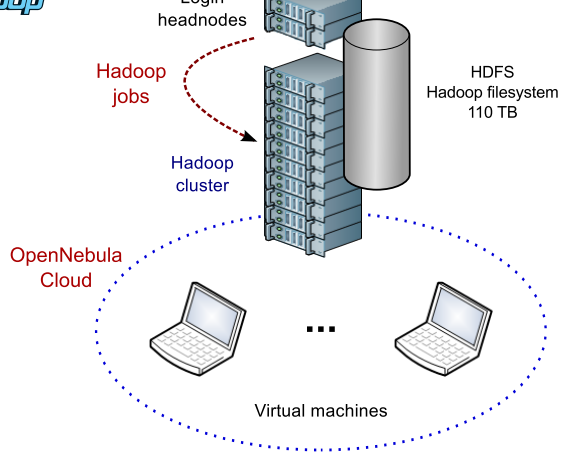




\section{How to deal with data?}

- With Cloud instances, if customized SW environment is required

- Integrated with the Kepler workflow orquestrator

- user-friendly interface 


\section{How to deal with data?}

- With Cloud instances, if customized SW environment is required

- Integrated with the Kepler workflow orquestrator

- user-friendly interface



- With workflows in the Hadoop cluster 


\section{How to deal with data?}

- With Cloud instances, if customized SW environment is required

- Integrated with the Kepler workflow orquestrator

- user-friendly interface

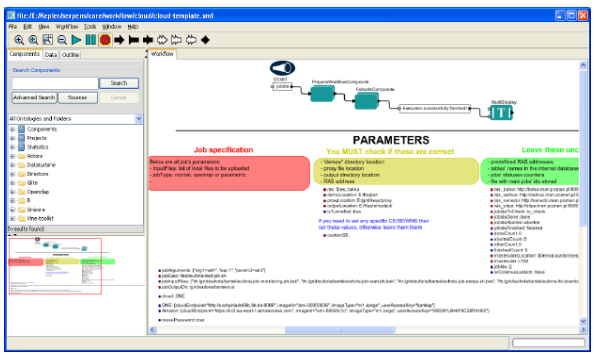

- With workflows in the Hadoop cluster 


\section{Processing data at LSDF}

- Experiments should be able to process data locally

$\Rightarrow$ help the users automate the workflows

- Allow tagging data and triggering
execution via DataBrowser
and tagged in DB
- used for zebrafish microscopy data

T. Jejkal et al., LAMBDA - The LSDF Execution Framework for

Data Intensive Applications, Proceedings PDP 2012. 


\section{Processing data at LSDF}

- Experiments should be able to process data locally $\Rightarrow$ help the users automate the workflows

- Allow tagging data and triggering execution via DataBrowser

- Data from finished workflows stored and tagged in DB

- used for zebrafish microscopy data

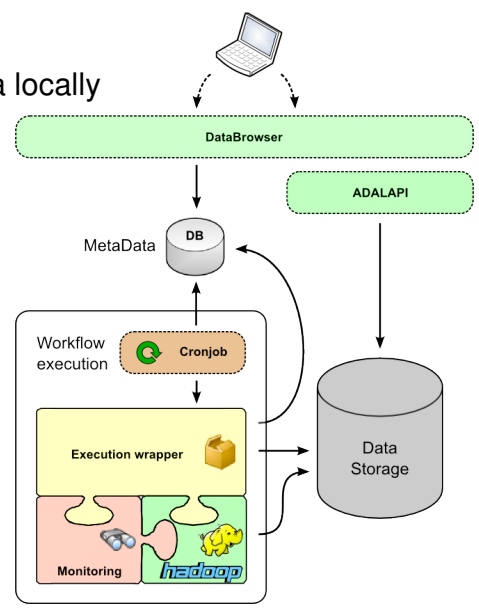

Data Intensive Applications, Proceedings PDP 2012. 


\section{Microscopy data analysis}

- Zebra-fish data sets

- Data imported with DataBrowser, meta-data added

- ImagingProjectGUI to define image analysis chain

- based on operators from different libraries (VTK, ITK, Matlab,...)

- creates sequence-XML, XML description of operators' chain

a User tags data for processing

- using DataBrowser

- associating sequence-XML

- Cron job starts execution based on tags

- projectProcessor

- Executed in Hadoop cluster

\section{- result-XML stored}

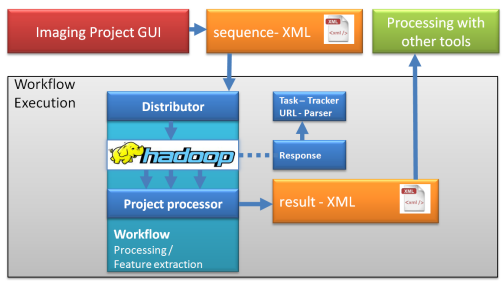

- Speedup of 60-120 depending on operators

1/O bound analysis

S. Pfeiffer, M. Mai, and J. Calliess, On the Computational Benefit of Tensor Separation for

High-Dimensional Discrete Convolutions, Multidimensional Systems and Signal Processing 31, 2010, pp. 1-25.

J.C. Otte, S. Pfeiffer, T. Schenker, and C. Schmidt, Concept of multidimensional image analysis with

flexible operators and cluster-computing, 6th European Molecular Imaging Meeting, Leiden, The Netherlands, 2011. 


\section{Microscopy data analysis}

- Zebra-fish data sets

- Data imported with DataBrowser, meta-data added

- ImagingProjectGUI to define image analysis chain

- based on operators from different libraries (VTK, ITK, Matlab,...)

- creates sequence-XML, XML description of operators' chain

- User tags data for processing

- using DataBrowser

- associating sequence-XML

- Cron job starts execution based on tags

- projectProcessor

- Executed in Hadoop cluster

- result-XML stored

- Speedup of 60-120 depending on operators

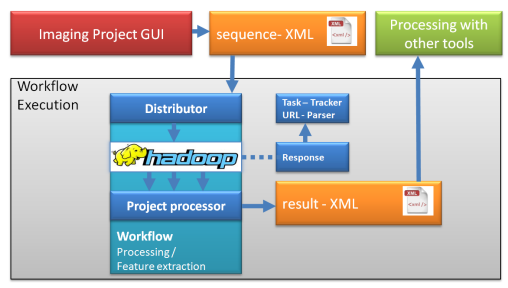

- I/O bound analysis

S. Pfeiffer, M. Mai, and J. Calliess, On the Computational Benefit of Tensor Separation for

High-Dimensional Discrete Convolutions, Multidimensional Systems and Signal Processing 31, 2010, pp. 1-25.

J.C. Otte, S. Pfeiffer, T. Schenker, and C. Schmidt, Concept of multidimensional image analysis with

flexible operators and cluster-computing, 6th European Molecular Imaging Meeting, Leiden, The Netherlands, 2011. 


\section{What's ahead?}

- Improved storage, network capacity: 6 PB in 2012

\section{Investigate and deploy new technologies}

- Data management system iRODS

- Object Storage

\section{- Additional communities being integrated}

- Meteorology and climate research ("archival" quality)

- Geophysics, seismology data

- KATRIN experiment, neutrino mass

- DARIAH, arts and humanities 


\section{What's ahead?}

- Improved storage, network capacity: 6 PB in 2012

\section{Investigate and deploy new technologies}

- Data management system iRODS

- Object Storage

- Additional communities being integrated

- Meteorology and climate research ("archival" quality)

- Geophysics, seismology data

- KATRIN experiment, neutrino mass

- DARIAH, arts and humanities 


\section{What's ahead?}

- Improved storage, network capacity: 6 PB in 2012

\section{Investigate and deploy new technologies}

- Data management system iRODS

- Object Storage

- Additional communities being integrated

- Meteorology and climate research ("archival" quality)

- Geophysics, seismology data

- KATRIN experiment, neutrino mass

- DARIAH, arts and humanities

\section{Project expansion}

- to offer more community tailored support 


\section{Concluding remarks}

- Infrastructure, 2 PB and storage services up and running

- First software tools available

- Experimental data being stored and processed

- Many scientific communities interested and getting involved

\section{Focus on users, added value services}

- Can't just "store files"

- Training for new tools, data management workflows

- Same problem at most (all?) research institutions

$\Rightarrow$ Open for new partnerships, international collaborations 
Thanks for listening!

\section{Questions?}

